\title{
Trauma infanto juvenil y psicopatología adulta: un estudio empírico
}

\author{
Kristina Weil $\mathbf{P}^{1}$, Ramón Florenzano $\mathbf{U}^{1,2}$, Verónica Vitriol $\mathbf{G}^{3}$, \\ Carlos Cruz $\mathbf{M}^{1}$, César Carvajal $\mathrm{A}^{\mathbf{1}}$, Claudio Fullerton $\mathrm{U}^{\mathbf{2}}$, \\ Carolina Muñiz D².
}

\author{
Child battering and adult \\ psychopathology: an empiric study
}

\begin{abstract}
Background: Childhood trauma and battering have been associated with adult psychopathology. Aim: To explore the relationship between childhood trauma, somatization, post traumatic stress disorder (PTSD), affective disorder and borderline personality disorder in hospitalized patients of four Chilean hospitals. Material and methods: Five hundred five patients were screened by a short seven item trauma recollection scale (70 from San Bernardo Hospital, 193 from Salvador Hospital, 97 from El Trabajador Hospital and 147 from Curico Hospital). A random sample of 85 cases was studied in depth using the CIDI 2.1, depression, PTSD and somatization scales, Inventory of Personality Organization (IPO) and the OQ 45.2 scale. Results: Forty five percent of patients did not report traumatic experiences, $38.4 \%$ recalled one or two events and $16.3 \%$ three or more traumatic experiences. The most remembered event was physical punishment (28.7\%), followed by traumatic separation from parents (27.1\%), alcohol and drug use by an adult at home (22\%) and presence of family violence (22\%). Thirty two percent of the 85 selected cases met CIDI criteria for affective disorder, $20 \%$ for post traumatic stress disorder and $11.8 \%$ for somatization disorder. There were statistically significant correlations between the frecuence of trauma and post traumatic stress disorder ( $p$ $<0.001$ ), as well as somatization and depressive disorder ( $p<0.007$ and 0.008 ). Conclusions: This study supports the concept that traumatic psychosocial environments during chilhood are a risk factor for diverse psychiatric syndromes during adulthood (Rev Méd Chile 2004; 132: - ).
\end{abstract}

(Key Words: Delayed onset post traumatic stress disorder; Personality disorders; Psychopathology; Stress disorders, post traumatic)

Recibido el 13 de mayo, 2004. Aceptado en versión corregida el 22 de septiembre, 2004. Trabajo apoyado por un subsidio del Fondo de Ayuda a la Investigación, Universidad de los Andes. Santiago de Chile.

${ }^{1}$ Universidad de los Andes, Santiago, Chile. ${ }^{2}$ Universidad de Chile, Hospital del Salvador, Santiago Chile. ${ }^{3}$ Hospital Regional de Curicó, Curicó, Chile.

Correspondencia a: Dra. Kristina Weil Parodi. Vitacura 5900, Of. 405 Santiago, Chile. E mail: kweil@uandes.cl 
$\mathrm{D}$ istintos estudios muestran una alta prevalencia de síntomas emocionales en poblaciones de pacientes consultantes en el nivel primario de atención de salud, principalmente de cuadros depresivos y ansiosos entre las mujeres y abuso de alcohol y drogas entre los hombres ${ }^{1-3}$. Existe evidencia creciente en la literatura, tanto nacional como internacional, acerca de las consecuencias psicopatológicas en la vida adulta de hechos traumáticos ocurridos en la niñez y preadolescencia. Por ejemplo, la asociación de somatización con experiencias traumáticas ha sido estudiada por distintos autores, en relación a trauma sexual $^{4,5}$, dolor en estrés postraumático ${ }^{6}$ y síntomas gastrointestinales ${ }^{7}$. En Chile, primero Numhauser et al, en estudios realizados en mujeres de Chiloé ${ }^{2}$ y luego nuestro grupo en poblaciones hospitalarias, han documentado esta asociación ${ }^{3}$. El número, tipo y gravedad de las experiencias traumáticas, así como la edad en que se producen, pudieran representar factores de riesgo importantes para la aparición de psicopatología en la adultez. Russek et al establecieron por su parte, en un estudio de 35 años de seguimiento, la asociación entre una menor aparición de síntomas físicos y psiquiátricos con la percepción positiva del cuidado parental ${ }^{8}$. Por otra parte, la estructura de personalidad pudiera influir en la aparición de síntomas somáticos y su relación con el trastorno de estrés postraumático también ha sido sugerida. El neuroticismo y la extroversión fueron identificados como factores de riesgo para este cuadro ${ }^{9}$. Spatz en una revisión de distintos autores encontró una relación entre niños tratados con negligencia en la infancia y distintos trastornos de personalidad, especialmente antisocial, borderline y narcisista ${ }^{10}$. Según Johnson, personas con antecedente de cuidado parental negligente 0 de abuso sexual infantil, son diagnosticadas cuatro veces más como portadoras de trastorno de personalidad durante la adultez temprana que la población general, si se controlan en el análisis estadístico factores asociados de los padres como edad, educación y presencia de trastornos psiquiátricos ${ }^{11}$. Por otra parte, se ha podido establecer el efecto anátomo-funcional del trauma psíquico sobre el cerebro: se produce una disminución del volumen hipocámpico, disminuye la integración hemisférica y se genera una hiperrespuesta del eje hipotálamo-hipófisis- suprarrenal ${ }^{12}$.
Varios autores han postulado una relación entre la expresión genética de la monoaminoxidasa, enzima de reconocida importancia en el metabolismo de los neurotransmisores ligados a cuadros psiquiátricos, y el maltrato infantil ${ }^{12,13}$.

Florenzano y cols, encontraron una mayor aproximación al riesgo relativo (Odds ratio) para presentar trastornos somatomorfos o de personalidad limítrofe, en pacientes con puntajes elevados en la escala de trauma de Marshall ${ }^{3,14}$.

Este trabajo intenta profundizar en el estudio de la relación entre trauma psíquico y patología psiquiátrica adulta, en pacientes hospitalizados en cuatro hospitales chilenos, midiendo psicopatología, problemas interpersonales y rol social, así como diagnósticos específicos: trastorno por estrés postraumático (TEPT), trastorno afectivo y de somatización. Además, se midieron indicadores de trastornos severos de personalidad, que se informan en otra publicación. Se administró, mediante entrevista directa una batería de instrumentos a un grupo de pacientes previamente seleccionados a través de un tamizaje con el cuestionario para trauma de Marshall, con el fin de analizar la relación entre trauma infantil y la presencia de psicopatología en la adultez.

\section{MATERIAL Y MÉTODO}

Todos los pacientes hospitalizados en los distintos servicios clínicos de los Hospitales Parroquial de San Bernardo, del Trabajador de Santiago, Regional de Curicó y del Salvador de Santiago, fueron entrevistados durante los días 14 y 15 de julio de 2003. Se excluyeron las pacientes con compromiso de conciencia o hipoacusia severa. Se obtuvieron las respectivas autorizaciones de los Comités de Ética de los hospitales participantes en el estudio, y los pacientes firmaron un formulario de consentimiento informado aprobado por dichos comités. Se consignaron datos socio-demográficos y los diagnósticos médicos de la ficha clínica. En la etapa de tamizaje se utilizó la escala de Trauma Infantil de Marshall, que registra el recuerdo anamnéstico de haber sufrido uno o más sucesos traumáticos antes de los 15 años de edad, entre siete propuestos (separación traumática del padre, madre o cuidador por más de un mes; experiencia de haber sufrido castigo físico importante; haber 
presenciado violencia física entre los padres 0 cuidador; abuso de alcohol o drogas por algún miembro de la familia; contacto sexual forzado con un pariente; daño físico después de haber sido castigado; contacto sexual forzado con un no familiar).

El total de pacientes evaluados fue de 505 pacientes (70 del Hospital Parroquial de San Bernardo, 97 del Hospital del Trabajador de Santiago, 145 del Hospital Regional de Curicó y 193 del Hospital del Salvador de Santiago). Del total de pacientes encuestados, se seleccionó una muestra aleatoria sistemática proporcional a la representación de cada centro hospitalario, que estuvo constituida por 85 casos. Estos pacientes fueron entrevistados nuevamente y se les administraron las sub-escalas para trastorno por estrés postraumático, trastorno afectivo y somatización del Composite International Diagnostic Interview (CIDI 2.1) de la Organización Mundial de la Salud.

Todos los instrumentos fueron administrados por estudiantes de medicina o psicología, quienes habían sido previamente capacitados.

Estadística. Los datos fueron codificados y analizados con el paquete computacional SPSS 11.4. Se consideró de valor estadísticamente significativo un $\mathrm{p}<0,05$.

\section{RESULTADOS}

Las poblaciones de pacientes de los cuatro hospitales resultaron comparables en cuanto a características sociodemográficas. La edad promedio de la muestra total fue de 49,4 años (DS 19,4). La distribución por género fue $48 \%$ de mujeres y $52 \%$ de hombres. El 58,2\% eran casados o con convivencia estable y $22,8 \%$ eran solteros. El 22,8\% tenían enseñanza media completa, $28,9 \%$ básica completa y 8,7\% no tenía educación. No hubo diferencias significativas para estado civil, religión ni escolaridad entre las poblaciones. Los servicios clínicos más representados fueron Cirugía y Medicina Interna con $26,5 \%$ y $23,2 \%$, respectivamente.

El número de eventos traumáticos para el total de la muestra estudiada se describe en la Tabla 1, así como su tipo en la Tabla 2. El 54,7\% de los pacientes refirió haber experimentado al menos un hecho traumático en su infancia. Del total de pacientes, $16,3 \%$ recordó al menos tres eventos traumáticos y $8,4 \%$ cuatro o más (Tabla 1 ).

En cuanto a la distribución por género, la distribución de hombres y mujeres con puntaje cero resultó muy similar, $45,4 \%$ y $45,2 \%$, respectivamente. El grupo con recuerdo de tres o más

\section{Tabla 1. N úmero de eventos traumáticos infantiles recordados por 505 pacientes hospitalizados en cuatro hospitales generales en Chile, julio 2003}

\begin{tabular}{|lrr|}
\hline Número de eventos & $\mathrm{n}$ & $\%$ \\
\hline Cero & 229 & 45,3 \\
Uno & 117 & 23,2 \\
Dos & 77 & 15,2 \\
Tres & 40 & 7,9 \\
Cuatro & 21 & 4,2 \\
Cinco & 16 & 3,2 \\
Seis & 5 & 1,0 \\
\hline
\end{tabular}

Tabla 2. Tipo de eventos traumáticos infantiles recordados por 505 pacientes hospitalizados en cuatro hospitales generales en Chile, julio 2003

\begin{tabular}{|lrr|}
\hline Tipo de evento & $\mathrm{n}$ & $\%$ \\
\hline Separación traumática del padre, madre o cuidador por más de un mes & 137 & 27,1 \\
Experiencia de haber sufrido castigo físico importante & 145 & 28,7 \\
Haber presenciado violencia física entre los padres o cuidador & 111 & 22,0 \\
Abuso de alcohol o drogas por miembro de la familia & 111 & 22,0 \\
Contacto sexual forzado con un pariente & 14 & 2,8 \\
Daño físico después de haber sido castigado & 47 & 9,3 \\
Contacto sexual forzado con un no familiar & 20 & 4,0 \\
\hline
\end{tabular}


eventos fue de $17,8 \%$ en las mujeres y $14,9 \%$ en los hombres.

Del total de 85 casos estudiados en la segunda fase, 27 (31,8\%) cumplieron con los criterios para trastorno afectivo, 17 (20\%) para TEPT, y 10 $(11,8 \%)$ para trastorno de somatización (Tabla 3).

Al comparar la distribución de los diagnósticos CIDI entre pacientes con y sin antecedente de trauma (Tabla 4) se encontró una asociación estadísticamente significativa entre la presencia de tres o más eventos traumáticos y TEPT $(64,3 \%$ versus $7 \%$; $p<0,0001$ ) como se observa en la Figura 1. Al comparar la distribución de los tres trastornos entre pacientes con y sin antecedente de trauma (Tabla 4), se puede constatar que entre aquellos casos con trastornos afectivos, 57,1\% recuerda traumatización infantil de tres o más tipos, en comparación a $25,6 \%$ de aquellos casos sin ese recuerdo. Las diferencias fueron estadísticamente significativas ( $p<0,008$ ). Respecto del trastorno de somatización, $42,9 \%$ de los casos presentaban tres o más traumas y sólo el 9,3\% no tenía ningún antecedente.

Tabla 3. Frecuencia de diagnósticos según CID I 2.1 en 85 pacientes hospitalizados de cuatro hospitales generales en C hile, julio 2003

\begin{tabular}{|lcc|}
\hline Diagnóstico & $\mathrm{n}$ & $\%$ \\
\hline Trastorno afectivo & 27 & 31,8 \\
Trastorno somatización & 10 & 11,8 \\
TEPT & 17 & 20,0 \\
\hline
\end{tabular}

\section{DisCUSIÓN}

El presente trabajo coincide con otros estudios nacionales $2,3,14$ e internacionales $5,6,9,11,15$ en cuanto a la asociación de trauma infantil y presencia de patología psiquiátrica en la edad adulta. Desde el punto de vista metodológico es necesario señalar que éste es un trabajo hecho a una extensa población hospitalaria, usándose en el tamizaje la escala de trauma descrita por Marshall, orientada al trauma civil, básicamente físico y sexual, que omite catástrofes naturales, cautiverio, guerras, etc. Es una escala corta de siete ítemes, que no discrimina dentro del trauma sexual ni físico sobre intensidad, edad de inicio, frecuencia y prolongación del abuso y muy someramente sobre la relación de parentesco con el agresor sexual, aspectos que han sido relacionados con la aparición de psicopatología y su pronóstico ${ }^{16-20}$. El realizar la entrevista cara a cara podría provocar un sesgo de deseabilidad social. A pesar de ello, encontramos un alto porcentaje de pacientes que recuerda algún hecho traumático (55\%), 16,3\% refirieron tres o más eventos de la escala de Marshall. Al estudiar en profundidad los 85 casos seleccionados al azar, 31,8\% cumplió con los criterios para Trastorno Afectivo de alguna índole, 20\% para TEPT y 11,8\% para Trastorno de Somatización. Se encontró una alta correlación entre antecedente de trauma infantil y trastorno de estrés en la vida adulta ( $p<0,0001)$. Hay que destacar cómo se eleva la frecuencia de diagnósticos al aumentar la cantidad de traumas recordados, con un incremento significativo sobre tres eventos recordados.

Tabla 4. Asociación entre antecedente anamnéstico de trauma infantil y presencia de trastornos afectivos, T EPT y trastorno de somatización en 85 pacientes hospitalizados en cuatro hospitales generales en Chile, julio 2003

\begin{tabular}{|lcccc|}
\hline $\begin{array}{l}\text { Puntuación } \\
\text { escala trauma }\end{array}$ & T. afectivo & TEPT & T. de somatización & N total \\
\hline 0 & & & & \\
$1-2$ & $25,6 \%$ & $7,0 \%$ & $9,3 \%$ & 43 \\
$\geq 3$ & $28,6 \%$ & $17,9 \%$ & $10,7 \%$ & 28 \\
$\mathrm{p}$ & $57,1 \%$ & $64,3 \%$ & $42,9 \%$ & 14 \\
\hline
\end{tabular}




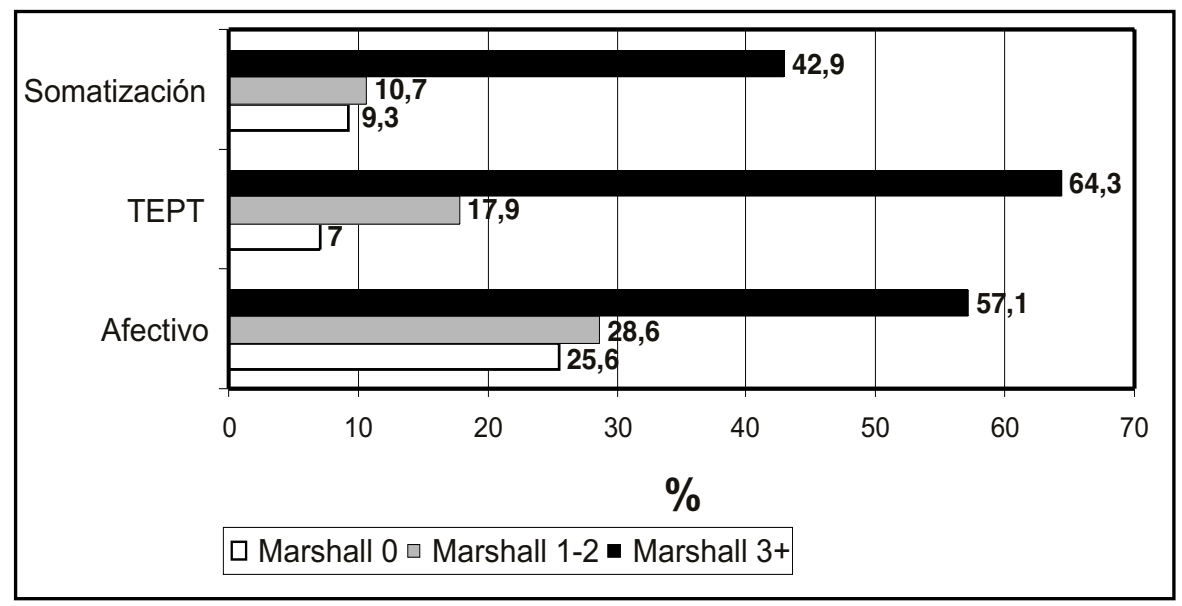

FIgURA 1. Frecuencia comparativa de TEPT, depresión y somatización en pacientes hospitalizados en cuatro hospitales chilenos, según puntaje en el test de screening de Marshall.

Destaca en este estudio una frecuencia de referencia de trauma sexual relativamente baja en relación a la literatura: 6,8\% versus $15 \%$ reportado internacionalmente ${ }^{21}$. Probablemente se relacione con la poca discriminación que hace la escala de Marshall en cuanto a qué considerar abuso sexual, pero pudiera tener que ver con una tendencia a callar dicha información, hecho estudiado por Vitriol en su trabajo con mujeres consultantes en un Servicio de Salud mental de Curicó («Referencia de Trauma Infantil en Psicopatología Adulta: estudio empírico en Mujeres consultantes en un Servicio de Salud Mental», tésis de magíster U. A Bello, Santiago de Chile 2002). Además encontramos frecuencia alta de TEPT (20\%), que se contradice con la baja frecuencia de diagnóstico de estos cuadros en la práctica clínica.

Aun considerando que se trata de un estudio con una casuística relativamente pequeña, podemos concluir que existe asociación estadísticamente significativa entre trauma infantil y algunos

\section{REFERENCIAS}

1. Florenzano R, Acuña J, Fuluerton C. Estudio comparativo de frecuencia y características de los trastornos emocionales en pacientes que consultan en el nivel primario de atención en Santiago de Chile. Rev Méd Chile 1998; 126: 397-405. trastornos psiquiátricos como somatización, trastorno afectivo y TEPT.

Este estudio es un corte transversal, por lo que no puede predicar causalidad: sólo podríamos concluir que los eventos psico-sociales traumáticos se relacionan causalmente si siguiéramos una cohorte prospectiva. Existe también el riesgo de un sesgo retrospectivo, al recordar más eventos quienes experimentan trastornos psicopatológicos.

Este estudio avala la evidencia clínica que señala que ambientes psico-sociales traumáticos en la niñez, pudieran producir diversos cuadros (especialmente TEPT) en la vida adulta. El trauma infantil pudiera tener una correlación inespecífica con cualquier patología mental, y no sólo con los cuadros aquí expuestos. Hemos abordado hipótesis específicas de relación con algunos cuadros, que complementaremos con el análisis de rasgos de trastornos graves de personalidad y la escala de OQ45.

2. Numhauser J, Schwartz G. Mujeres Sobrepasadas. Actas del Congreso Mundial para la Salud Mental, Agosto 1999, Santiago de Chile.

3. Florenzano R, Weil K, Acuña J, Fuluerton C, Cruz C, MuÑIZ C, Leighton C. Personalidad Limítrofe, Somatización, Trauma y Violencia Infantil: un nuevo estudio con muestra mayor. Revista Chilena Salud Mental 2002; 149-55. 
4. Mayou $R$, Sharpe M. Treating medically unexplained physical symptoms. Br Med J 1997; 315: 561.

5. LESERMAN J. Sexual and physical abuse history in Gastroenterology: how the types of abuse influence the health. Psych Med 1996; 58: 4-15.

6. Schreiber S, GaLaI-Gat T. Uncontrolled pain after injury as central trauma in the post-traumatic stress disorder. Pain 1993; 54: 107-10.

7. Walker E, Katon W. Sexual agression history in patients with irritable colon or intestinal inflammatory disease. Am J Psychiatry 1993; 150: 15026.

8. Russer L, Schwartz G, Bell I. Positive perception of the parents' care related with fewer somatic and psychiatric symptoms. Psych Med 1998; 60: 654-7.

9. BReSLAu N, Davis G, ANDRESKY P. Risk factors for post-traumatic stress disorder related with traumatic events: a prospective analysis. Am J Psychiatry 1995; 152: 529-35.

10. Spatz C. Childhood victimization and the development of personality disorders. Arch Gen Psychiatry 1999; Vol 56.

11. Johnson J, Cohen P. Childhood maltreatment increases risk for personality disorder during early adulthood. Arch Gen Psychiatry 1999; 56: 600-6.

12. Weiss E, Longhurst J, MCguiRe C. Childhood sexual abuse a risk factor for depression in women: Psychosocial and Neurobiological Correlates. Am J Psychiatry 1999; 156: 816-28.
13. Gold PW, Goodwin FK, Chrousos GP. Clinical and Biochemical Manifestations in Depression in relation to Neurobiology of Stress. N Engl J Med 1988; 319: 413-20.

14. Florenzano R, Fulerton C, Acuña J, Escalona R. Somatización: aspectos teóricos, epidemiológicos y clínicos. Rev Chil Neuropsiquiatría 2002; 47-54.

15. WaLKer E, KatON W. Psychiatric and Medical Symptoms in Women with Sexual Abuse History during Childhood. Psych Med 1992; 54: 658-64.

16. Mc LEAN L, GALOP R. Implication of Childhood Sexual Abuse for adult Borderline Personality Disorder and Complex Post-traumatic Stress Disorder. Am J Psychiatry 2003; 160: 369-71.

17. Pribor EF, Dinwiddie SH. Psychiatric correlate of Incest in Childhood. Am J Psychiatry 1992; 149: 52-6.

18. Katrin WJ, Hanson J. Medical and Psychiatric Symptoms in women with History of Childhood Abuse. Psychosom Med 1992; 54: 658-64.

19. BIFULCO A, Brown GW, AdLER Z. Early Sexual Abuse and Clinical Depression in Adult Life. Br J Psychiatry 1991; 159: 115-22.

20. Tsai M, Feldman-Summers S, Edgar M. Childhood Molestation: variables related to differential impacts on psychosexual functioning in adult women. J Abnorm Psychol 1979; 88: 407-17.

21. FinKelHOR K. The Sexual Abuse of children: Current Research rewied. Psychiatr Annals 1987; 17: 233-24. 